\section{POS1199 CLINICAL MANIFESTATIONS OF SARS-CoV2 INFECTIONS IN CHILDREN AND ADOLESCENTS WITH RHEUMATIC AND MUSCULUSKELETAL DISEASES - SURVEY DATA FROM GERMANY}

C. Sengler ${ }^{1}$, S. Eulert ${ }^{2}$, M. Niewerth ${ }^{2}$, T. Kallinich ${ }^{3}$, H. Wittkowski ${ }^{4}$, H. Girschick ${ }^{5}$

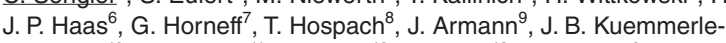
Deschner ${ }^{10}$, J. Brunner ${ }^{11}$, M. Borte ${ }^{12}$, R. Hühn ${ }^{13}$, K. Minden ${ }^{2}$, A. Klein ${ }^{7}{ }^{1}$ Gernan Rheumatism Research Center Berlin, Epidemiology Unit, Berlin, Germany; ${ }^{2}$ German Rheumatism Research Center Berlin, Epidemiology Unit, Berlin, Germany; ${ }^{3}$ Charité - Universitätsmedizin Berlin, Department of Pediatrics, Berlin, Germany: ${ }^{4}$ Universitätsklinikum Münster - UKM, Klinik für Kinder- und Jugendmedizin, Münster, Germany; ${ }^{5}$ Krankenhaus im Friedrichshain, Klinik für Kinder- und Jugendmedizin, Berlin, Germany; ${ }^{6}$ Deutsches Zentrum für Kinderund Jugendrheumatologie, Kinder- und Jugendrheumatologie, GarmischPartenkirchen, Germany; ${ }^{7}$ Allgemeine Kinder- und Jugendmedizin - Asklepios Klinik Sankt Augustin, Kinderrheumatologie, Sankt Augustin, Germany; ${ }^{8}$ Olgahospital and Women's Clinic, Pädiatrie 2 - Allgemeine und spezielle Pädiatrie, Stuttgart, Germany; ${ }^{9}$ University Hospital Carl Gustav Carus Dresden, Kinik und Poliklinik für Kinder- und Jugendmedizin, Dresden, Germany; ${ }^{10}$ Tübingen University Hospital, Department of Pediatrics, Tübingen, Germany; ${ }^{11}$ Tirol Kliniken GmbH, Department für Kinder- und Jugendheilkunde, Innsbruck, Austria: ${ }^{12}$ Klinikum St. Georg, Leipzig, Klinik für Kinder- und Jugendmedizin, Leipzig, Germany; ${ }^{13}$ Universitätsklinikum Halle (Saale), Klinik für Kinder- und Jugendmedizin, Halle (Saale), Germany

Background: Although children and adolescents are less likely to develop COVID-19 and generally show milder disease courses, it is unclear what impact the SARS-CoV2 infection has on children and adolescents with rheumatic and musculoskeletal disease (RMD). Due to their underlying disease as well as therapeutic immunosuppression these patients may be at higher risk of being more severely affected by SARS-CoV2. Furthermore, SARS-CoV2 infection might trigger a flare of the underlying disease.

Objectives: To evaluate clinical characteristics and disease course of COVID-19 in children and adolescents with RMD and to analyze possible effects of SARSCoV2 infection on the underlying disease under different therapeutic regimens. Methods: Data from juvenile patients with RMD recorded via the SARS-CoV2 questionnaire within the National Pediatric Rheumatology Database and the registry for hospitalized children and adolescents with COVID-19 of the German Society for Pediatric Infectious Diseases were analyzed. In addition to age, sex and diagnosis, information was collected about the date and method of a positive SARS-CoV2 testing, reason for testing, on clinical manifestations, disease course, treatment and outcome of COVID-19, on drug therapy at the time of virus detection, on disease activity (NRS $0-10,0=$ best) of the underlying disease at the last visit before and after the SARS-CoV2 infection.

Results: From April $17^{\text {th }} 2020$ until January $25^{\text {th }} 2021$, data of 67 patients with RMD and confirmed SARS-CoV2 infection were collected. Mean age was 13.5 \pm 3.9 years with equal sex distribution. The majority of patients were diagnosed with juvenile idiopathic arthritis (JIA, 64\%), 12 (18\%) patients had an autoinflammatory disease (FMF, CAPS, PFAPA, TRAPS) and $5(7 \%)$ a connective tissue disease. Fifty-two patients $(78 \%)$ were treated with a disease modifying antirheumatic drug (DMARD), 39\% with a biological DMARD and $9 \%$ systemic glucocorticoids at the time of SARS-CoV-2 infection. Nineteen patients $(28 \%)$ were tested for SARS-CoV-2 because of typical symptoms, the majority $(67 \%)$ because of contact to an infected person. PCR was used most often (in $60 \%$ ).

52 patients $(78 \%)$ developed symptoms of COVID-19, 15 patients remained asymptomatic. The most common symptom of COVID-19 was rhinitis $(42 \%)$ and fever $(38 \%)$, followed by fatigue $(34 \%)$, taste/smell disorder $(33 \%)$, sore throat $(27 \%)$ and cough $(23 \%)$

Disease severity was graded as mild in 44 of $52(85 \%)$ symptomatic patients, only two patients were hospitalized, one of whom required intensive care and died of cardiorespiratory failure 3 days after symptom onset. In 22 of $26(85 \%)$ SARS-CoV2-positive patients, no relevant increase in disease activity (difference in NRS $\leq 1$ before/after infection) of the underlying disease was observed 31 days after symptom onset (median, IQR 17-52 days). One patient, who had paused tocilizumab for 2 doses, experienced a flare of his seronegative polyarthritis 2 months after asymptomatic SARS-CoV-2 infection.

Conclusion: In our cohort, the clinical picture of COVID-19 in children and adolescents with RMD was similar to that of healthy peers. The majority of patients showed mild disease course with good outcome under various medications, however, one patient with a severe course of COVID-19 died. In addition, SARSCoV2 infection does not appear to have a relevant impact on the underlying disease activity, whereas discontinuation of therapy might pose a risk of flare. Disclosure of Interests: None declared.

DOI: 10.1136/annrheumdis-2021-eular. 1875

\section{POS1200 IL-6 HIGHER LEVEL AFTER TOCILIZUMAB TREATMENT AS A PREDICTOR OF NOT-SURVIVAL IN COVID19 SEVERE PNEUMONIA:THE EXPERIENCE OF THE COVIBAS-STUDY}

M. Gilio ${ }^{1}$, S. B. Morella ${ }^{1}$, M. A. Purgatorio ${ }^{2}$, D. Palazzo ${ }^{1}$, M. Frontuto ${ }^{1}$,

A. Erezanu ${ }^{1}$, G. Mastroberti', A. Linzalone ${ }^{3}$, G. Pietromatera ${ }^{3}$, A. Fineo ${ }^{3}$, M. Onofrio ${ }^{3}$, G. Casciano ${ }^{3}$, G. De Stefano ${ }^{2}{ }^{1}$ San Carlo Hospital, Infectious Disease, Potenza, Italy; ${ }^{2}$ San Carlo Hospital and Madonna delle Grazie Hospital, Infectious Disease, Matera, Italy; ${ }^{3}$ Madonna delle Grazie Hospital, Infectious Disease, Matera, Italy

Background: Cytokine release storm is the most fearsome complication associated with worse outcomes in COVID-19 patients with severe acute respiratory failure. In COVID-19 patients, interleukin-6 (IL-6) levels are significantly elevated. Blocking IL-6 preliminarily resulted in the improvement of this hyperinflammatory state.

Objectives: To define if the serum level of IL- 6 early tested after tocilizumab treatment could be useful to distinguish non-survivors from survivors in patients with severe respiratory failure due to COVID-19 pneumonia.

Methods: This prospective cohort study was conducted at two referral hospitals for management of COVID-19 in Basilicata region, Italy: San Carlo Hospital, Potenza and Madonna delle Grazie Hospital, Matera. All consecutive patients with COVID-19 confirmed by RT-PCR testing of a naso-oropharyngeal swab. The enrollement period was from the 23rd of February to the 15th of August. 37 patients between all 124 underwent tocilizumab $8 \mathrm{mg} / \mathrm{kg}$ intravenously at baseline and after $24 \mathrm{~h}$. All patients were tested for serum IL-6, 24 hours before and 72 hours after tocilizumab infusion. Comparisons between survivors (Sv) and nonsurvivors (NSv) were performed.

Results: 21 patients were discharged, while 16 patients died. The main clinical or laboratory differences $(p<0.001)$ between the two groups at baseline were mean diseases duration before ward admission and serum troponine levels. IL- 6 was not different at baseline $(p=0.01)$, while 72 hours post-tocilizumab IL-6 serum levels were significantly higher in non-survivors than in survivors [(Sv $21.1 \%) v s(N S v 64 \%) p<0.001)]$. Serum IL-6 post-tocilizumab could be considered a good predictor to discriminate Sv from NSv. At multivariate stepwise analysis including disease duration at admission, baseline serum troponine levels and IL-6 post TCZ) were independent predictors of not survival

Conclusion: Our data confirm the results of Quartuccio et al. In fact the highest burden of inflammation may be revealed by IL- 6 levels after tocilizumab. Therefore repeated measurement of the serum level of IL- 6 early after tocilizumab could be useful to distinguish non-survivors from survivors and support the choice of deeper targeting IL-6 in COVID-19 pneumonia.

\section{REFERENCES:}

[1] Mehta P, McAuley DF, Brown M, et al. COVID-19: consider cytokine storm syndromes and immunosuppression. Lancet. 2020; 395(10229): 1033- 1034

[2] Toniati P, Piva S, Cattalini M, et al. Tocilizumab for the treatment of severe COVID-19 pneumonia with hyperinflammatory syndrome and acute respiratory failure: a single center study of 100 patients in Brescia, Italy. Autoimmun Rev. 2020; 19:102568

[3] McGonagle D, Sharif K, O'Regan A, Bridgewood C. The role of cytokines including interleukin 6 in COVID19 induced pneumonia and macrophage activation syndrome-like disease. Autoimmun Rev. 2020; 19(6):102537.

[4] Chakraborty C, Sharma AR, Bhattacharya M, Sharma G, Lee SS, Agoramoorthy G. COVID19: consider IL6 receptor antagonist for the therapy of cytokine storm syndrome in SARS CoV2 infected patients. J Med Virol 2020

[5] Quartuccio L, Sonaglia A, Pecori D,et al. Higher levels of IL-6 early after tocilizumab distinguish survivors from nonsurvivors in COVID-19 pneumonia: A possible indication for deeper targeting of IL-6. J Med Virol.2020;92:2852-2856

Disclosure of Interests: None declared

DOI: 10.1136/annrheumdis-2021-eular.1902 